\title{
Berichte über die Tätigkeit der zur Erforschung der Cholera im Jahre 1883 nach Ägypten und Indien entsandten Kommission ${ }^{1}$ )
}

\author{
an S. Exzellenz den Staatssekretär des Innern Herrn \\ Staatsminister von Bötticher \\ erstattet \\ vom Geheimen Regierungsrat Dr. R. Koch.
}

A lexandrien, den 17. September 1883.

Ew. Exzellenz beehre ich mich über den Fortgang der Untersuchungen zur Erforschung der Cholera*) ganz gehorsamst nachstehenden Bericht zu erstatten.

Da die Cholera beim Eintreffen der Kommission in Ägypten bereits in schneller Abnahme begriffen war, so ließ sich von vornherein nicht erwarten, in diesem Lande das für den ganzen Umfang der Untersuchung erforderliche Material zu gewinnen; da außerdem die Zeit des Erlöschens einer Epidemie am wenigsten für die ätiologische Erforschung derselben geeignet ist, so ging der ursprüngliche Plan dahin, in Ägypten die nötigen Vorstudien zu machen und diese, wenn die Epidemie sich nach Syrien ausbreiten würde, in solchen Orten, welche von der Cholera erst eben befallen wären und für die Untersuchungen einen günstigen Boden geliefert hätten, zu verwerten.

Der erste Teil dieses Planes hat sich bisher allen Wünschen entsprechend ausführen lassen; denn die Kommission hat während ihres Aufenthaltes in Alexandrien noch hinglänglich Gelegenheit gefunden, das zum Vorstudium notwendige Material zu sammeln. Daß dies gelungen ist, verdanke ich weniger den Bemühungen der ägyptischen Behörden, welche allerdings vielversprechende Verheißungen gemacht und gleich am ersten Tage auch eine Choleraleiche zur Verfügung gestellt hatten, als dem Entgegenkommen der Ärzte des griechischen Hospitals, welche dadurch, daß sie Arbeitsräume und alle ins Hospital gelangenden Cholerakranken, sowie Choleraleichen zur Verfügung stellten, die Zwecke der Expedition in wirksamster Weise förderten. Anfangs hatte sich die Kommission in zwei zu ebener Erde und nebeneinander gelegenen hellen Zimmern des Hospitals eingerichtet. In dem einen Raume wurden die mikroskopischen Arbeiten, im zweiten die Kulturversuche ausgeführt. Die Versuchstiere waren in beiden untergebracht. Als aber die Zahl der Versuchstiere zunahm und es auch zu gefährlich erschien, in denselben Räumen, in welchen man sich fast den ganzen Tag aufhalten mußte, mit den Infektionsstoffen zu manipulieren, wurden die Versuchstiere in einen vollständig abgetrennten Raum des alten Hospitals gebracht und dort die Infektionsversuche angestellt.

1) Aus Arbeiten aus dem Kaiserl. Gesundheitsamte, 1887, Bd. III. Verlag von Julius Springer, Berlin.

*) Den ersten, bisher unveröffentlichten Bericht siehe in der zweiten Hälfte dieses Bandes.

D. Herausgeber.

Koch, Gesammelte Werke.

46 
Das bisher zur Untersuchung gelangte Material stammt von 12 an Cholera Erkrankten und von 10 Choleraleichen.

Von den Kranken wurden $9 \mathrm{im}$ griechischen, 2 im deutschen und 1 im arabischen Hospital beobachtet. Die Krankheitssymptome entsprachen in allen Fällen in jeder Beziehung denjenigen der echten asiatischen Cholera. Es wurden Proben von Blut dieser Kranken, vom Erbrochenen und von den Dejektionen derselben entnommen und untersucht. Da sich sehr bald herausstellte, daß das Blut frei von Mikroorganismen und auch die erbrochenen Massen verhältnismäßig arm daran waren, aber die Dejektionen bedeutende Mengen von Mikroorganismen enthielten, so wurden vorwiegend diese zu den Ansteckungsversuchen an Tieren benutzt.

Obwohl die Zahl der sezierten Leichen nur gering ist, so hat es.doch der Zufall so gefügt, daß dieselben ein für Orientierungszwiecke höchst wertvolles Material bieten. Es sind die verschiedensten Nationalitäten darunter vertreten (3 Nubier, 2 DeutschÖsterreicher, 4 Griechen, 1 Türke), verschiedene Altersstufen (2 Kinder, 2 im Alter über 60 Jahre, die übrigen zwischen 20 und 25 Jahre alt) und Fälle verschiedener Krankheitsdauer. Am wichtigsten ist jedoch, daß die Leichen meistens unmittelbar nach dem Tode oder doch wenige Stunden später seziert werden konnten. Die Veränderungen, welche in den Organen und ganz besonders frühzeitig im Darm durch die Fäulnis bedingt werden, und welche die mikroskopische Untersuchung dieser Organe im höchsten Grade erschweren und meistens illusorisch machen, wurden unter diesen Verhältnissen mit Sicherheit ausgeschlossen. Ich möchte gerade auf diesen Umstand um so größeres Gewicht legen, als es an anderen Orten kaum zu ermöglichen sein wird, ein für die mikroskopische Untersuchung so geeignetes Material zu gewinnen. Auch der Leichenbefund ließ ebenso wie die Krankheitssymptome keinen Zweifel, daß es sich hier um die echte Cholera handelt und nicht, wie von mehreren Seiten anfangs behauptet wurde, um choleraähnliche, sogenannte choleriforme oder choleroide Krankheiten.

Im Blute, sowie in den Organen, welche bei anderen Infektionskrankheiten gewöhnlich der Sitz der Mikroparasiten sind, nämlich in den Lungen, Milz, Nieren, Leber, konnten keine organisierten Infektionsstoffe nachgewiesen werden. Einige Male fanden sich in der Lunge Bakterien, welche jedoch, wie sich aus dem Verhalten ihrer Form und ihrer Lagerung ergab, mit dem eigentlichen Krankheitsprozeß nichts zu tun hatten, sondern durch die Aspiration des erbrochenen Mageninhaltes in die Lunge gelangt waren.

Im Inhalte des Darmes kamen ebenso wie in den Dejektionen der Cholerakranken außerordentlich viele und den verschiedensten Arten angehörige Mikroorganismen vor. Keine derselben trat in überwiegender Menge hervor. Auch bot keine sonstige Anzeichen, welche auf eine Beziehung zum Krankheitsprozeß hätte schließen lassen.

Dagegen ergab der Darm selbst ein sehr wichtiges Resultat. Es fanden sich nämlich mit Ausnahme eines Falles, welcher mehrere Wochen nach dem Überstehen der Cholera an einer Nachkrankheit tödlich geendet hatte, in allen übrigen eine bestimmte Art von Bakterien in den Wandungen des Darmes. Diese Bakterien sind stäbchenförmig und gehören also zu dẹn Bazillen, sie kommen in Größe und Gestalt den bei der Rotzkrankheit gefundenen Bazillen am nächsten. In denjenigen Fällen, in denen der Darm mikroskopisch die geringsten Veränderungen zeigte, waren die Bazillen in die schlauchförmigen Drüsen der Darmschleimhaut eingedrungen und hatten daselbst, wie die Erweiterung des Lumens der Drüse und die Ansammlung von mehrkernigen Rundzellen im Innern der Drüse beweisen, einen erheblichen Reiz ausgeübt. Vielfach hatten sich die Bazillen auch hinter dem Epithel der Drüse einen Weg gebahnt und waren zwischen Epithel und Drüsenmembran hineingewuchert. Außerdem hatten sich die Bazillen in reichlicher Menge an der Oberfläche der Darmzotten angesiedelt und waren oft in das Gewebe derselben 
eingedrungen. In den schweren mit blutiger Infiltration der Darmschleimhaut verlaufenden Fällen fanden sich die Bazillen in sehr großer Anzahl, und sie beschränkten sich dann auch nicht allein auf die Invasion der schlauchförmigen Drüsen, sondern gingen in das umgebende Gewebe, in die tieferen Schichten der Schleimhaut und stellenweise sogar bis zur Muskelhaut des Darmes. Auch die Darmzotten waren in solchen Fällen reichlich von Bazillen durchsetzt. Der Hauptsitz dieser Veränderungen befindet sich im unteren Teile des Dünndarmes. Wenn dieser Befund nicht an ganz frischen Leichen gewonnen wäre, dann hätte man ihn wenig oder gar nicht verwerten können, weil der Einfluß der Fäulnis imstande ist, ähnliche Bakterienvegetation im Darme zu veranlassen. Aus diesem Grunde hatte ich auch darauf, daß ich bereits vor einem Jahre im Choleradarm, welchen ich direkt aus Indien erhalten hatte, dieselben Bazillen und in derselben Anordnung wie jetzt in den ägyptischen Cholerafällen gefunden, früher keinen Wert legen können, weil immer an eine Komplikation mit postmortalen Fäulnisvorgängen gedacht werden mußte. Jetzt gewinnt aber dieser frühere Befund, welcher am Darme von vier verschiedenen indischen Choleraleichen gemacht wurde, außerordentlich an Wert, da sich nunmehr ein durch Fäulniserscheinungen bedingter Irrtum sicher ausschließen läßt. Nicht unwichtig ist auch, daß durch die Übereinstimmung in dem Verhalten des Darmes bei der indischen und der ägyptischen Cholera ein weiterer Beweis für die Identität beider Krankheiten gewonnen wird.

Die Zahl der zur Untersuchung gelangten Choleraleichen ist allerdings nur eine verhältnismäßig geringe, da aber die Bazillen in allen frischen Cholerafällen angetroffen wurden, dagegen in dem einen nach Ablauf des Choleraprozesses untersuchten Falle und bei mehreren anderen, an anderweitigen Krankhieiten Gestorbenen und vergleichsweise ebenfalls daraufhin untersuchten Fällen vermißt wurden, so kann kein Zweifel darüber sein, daß sie in irgendeiner Beziehung zu dem Choleraprozesse stehen. Jedoch ist aus dem Zusammentreffen des letzteren mit dem Vorkommen von Bazillen in der Darmschleimhaut noch nicht zu schließen, daß die Bazillen die Ursache der Cholera seien. Es könnte auch umgekehrt sein und es ließe sich ebensogut annehmen, daß der Choleraprozeß derartige Zerstörungen in der Darmschleimhaut hervorruft, daß unter den vielen, im Darm beständig schmarotzenden Bakterien einer bestimmten Bazillenart das Eindringen in die Gewebe der Darmschleimhaut ermöglicht wird.

Welche von diesen beiden Annahmen die richtige ist, ob der. Infektionsprozeß oder ob die Bakterieninvasion das Primäre ist, das läßt sich nur dadurch entscheiden, daß man versucht, die Bakterien aus den erkrankten Geweben zu isolieren, sie in Reinkulturen zu züchten und dann durch Infektionsversuche an Tieren die Krankheit zu reproduzieren. $\mathrm{Zu}$ diesem $Z_{w}$ ecke ist es vor allem notwendig, solche Tiere zur Verfügung zu haben, welche für den fraglichen Infektionsstoff empfänglich sind. Nun ist es aber bisher trotz aller Bemühungen nicht in unanfechtbarer Weise gelungen, Tiere cholerakrank zu machen. Man hat an Kaninchen, Meerschweinchen, Hunden, Katzen, Affen, Schweinen, Ratten usw. vielfach experimentiert, aber immer erfolglos. Die einzigen Angaben, welche in dieser Beziehung Beachtung verdienen, sind von $\mathrm{Th}$ ie $\mathrm{r} \mathrm{s} \mathrm{ch}$ gemacht, welcher nach Verfütterung von Choleradarm eine Anzahl von Mäusen am Durchfall erkranken und sterben sah. Dieser Versuch ist von zuverlässigen Experimentatoren wie $\mathrm{B}$ u rdon-S a n d e r s on bestätigt, von anderen allerdings bestritten worden. Immerhin war es, da das Auffinden einer für Cholera empfänglichen Tierspezies von der größten Wichtigkeit ist, notwendig, diese Versuche zu wiederholen. Zu diesem Zwecke wurden, weil es sehr unwahrscheinlich war, daß die erforderliche Anzahl Mäuse in Alexandrien bald zu beschaffen sein würde, schon von Berlin 50 Mäuse mitgeführt und mit diesen die Infektionsversuche sofort begonnen. Außerdem wurden aber auch noch Affen, 
welche für einige menschliche Infektionskrankheiten, wie Pocken und Recurrens, die einzig empfängliche Tierspezies sind, gleichfalls für diese Versuche verwendet. Schließlich wurden auch einige Hunde und Hühner zu infizieren versucht. Aber trotz aller Bemühungen sind diese Versuche bislang gänzlich resultatlos geblieben. Es wurden die verschiedensten Proben von Erbrochenem, von Choleradejektionen und vom Darminhalt der Choleraleichen teils frisch, teils nachdem sie längere Zeit in kaltem oder warmem Raume gestanden hatten, teils getrocknet an die Tiere verfüttert. Aber es traten niemals choleraartige Erscheinungen ein, die Tiere blieben im Gegenteil vollkommen gesund. Es waren ferner von den im Darminhalt und in den Darmwandungen vorkommenden Bazillen Reinkulturen gemacht, und auch mit diesen sind Fütterungsversuche, zum Teil auch Impfungen ausgeführt. Einzelne dieser Reinkulturen bewirkten septische Erkrankungen, wenn sie verimpft wurden, aber mit keiner konnte Cholera erzeugt werden. Daß in den Dejektionen der Cholerakranken der Krankheitsstoff in wirksamer Form sehr oft enthalten sein muß, das ist durch vielfache Erfahrungen, namentlich durch das häufige Erkranken von Wäscherinnen, welche mit Dejektionen beschmutzte Cholerawäsche zu waschen hatten, bewiesen. Auch im griechischen Hospital ist in der jetzigen Epidemie ein solcher Fall vorgekommen und eine Wäscherin, welche ausschließlich die Cholerawäsche zu besorgen hatte, an Cholera erkrankt. Es ist demnach wohl als sicher anzunehmen, daß in den zahlreichen zur Verwendung gekommenen Proben mindestens einige den Infektionsstoff enthalten haben. Wenn dennoch keine Resultate erzielt wurden, so kann es daran gelegen haben, daß die zu den Versuchen dienenden Tierarten für die Cholera überhaupt unempfänglich sind, oder daß noch nicht der richtige Modus der Infektion gefunden wurde. Sowohl in der einen wie in der anderen Richtung sollen die Versuche fortgesetzt und modifiziert werden, doch ist wenig Aussicht vorhanden, daß auf diesem Wege mit dem jetzt zur Verfügung stehenden Material etwas erreicht wird. Denn es ist nicht sehr wahrscheinlich, daß allein in jenen Umständen der Grund für das Mißlingen der Infektionsversuche zu suchen ist. Es gibt noch eine dritte Erklärung, für deren Richtigkeit sehr vieles spricht. In einem von der Cholera befallenen Orte hört bekanntlich die Krankheit auf, lange bevor alle Individuen durchseucht sind, und obwohl der Krankheitsstoff schließlich in großer Menge über den ganzen Ort ausgestreut ist, so erkranken doch immer weniger Menschen, und die Epidemie erlischt mitten'unter vielen für die Ansteckung empfänglichen Individuen. Diese Erscheinung ist nur durch die Annahme erklärbar, daß gegen Ende der Epidemie der Infektionsstoff an Wirksamkeit einbüßt oder wenigstens unsicher in seiner Wirkung wird. Wenn nun aber selbst die Menschen gegen Ende der Epidemie auf den Cholerainfektionsstoff nicht mehr reagieren, dann läßt sich nicht erwarten, daß dies bei Versuchstieren der Fall sein soll, über deren Empfänglichkeit für Cholera man noch nichts weiß. Für unsere Versuche standen uns nun aber nur solche Objekte zur Verfügung, welche am Ende der Epidemie gesammelt wurden, und deren Unwirksamkeit mehr oder weniger vorausgesetzt werden mußte. Es ist immerhin möglich, daß unter günstigeren Verhältnissen, d. h. zu Anfang einer Epidemie, die Infektion von Tieren gelingt und damit auch sofort zu erfahren ist, ob die in der Darmschleimhaut von mir nachgewiesenen Bazillen die eigentliche Ursache der Cholera bilden.

Soweit nun auch die von der Kommission bisher erhaltenen Resultate von der vollständigen Lösung der Aufgabe noch entfernt sind, und so wenig sie zu einer praktischen Verwertung in der Bekämpfung der Cholera geeignet sind, so dürfen sie in Anbetracht der ungünstigen Verhältnisse und der kurzen Zeit der Untersuchung dennoch als günstige gelten. Sie entsprechen vollkommen dem ursprünglichen Zwecke der Orientierung und gehen insofern noch darüber hinaus, als durch den konstanten Befund von 
charakteristischen Mikroorganismen der ersten Bedingung, welche bei der Erforschung einer Infektionskrankheit zu erfüllen ist, Genüge geleistet und damit der weiteren Forschung ein bestimmtes Ziel gesteckt ist.

Ew. Exzellenz wollen aus der gehorsamst gegebenen Darlegung hochgeneigtest entnehmen, daß die Kommission in der Lösung der ihr gestellten Aufgabe in Alexandrien nicht weiter zu gelangen vermag, als bisher geschehen ist. Es würde nunmehr die Frage an die Kommission herantreten, ob nicht an einem anderen von der Cholera heimgesuchten Orte Ägyptens die Untersuchungen fortzusetzen sind. Dem stellen sich aber unüberwindliche Hindernisse entgegen. In allen größeren Städten Ägyptens ist die Cholera bereits ganz erloschen. Nur in den Dörfern Oberägyptens macht die Epidemie noch einige Fortschritte. Durch Vermittelung des deutschen Konsulats wurde deswegen eine Anfrage an den Ministerpräsidenten Chérif Pascha gerichtet, ob es möglich sei, in den von der Cholera befallenen Dörfern Material für die Untersuchung zu gewinnen. Die hierauf erteilte telegraphische Antwort lautete aber folgendermaßen: Je ne puis conseiller à Monsieur le Dr. K o c h de se rendre dans les villages pour faire des autopsies, il est même de mon devoir de l'en dissuader, car elles pourraient donner lieu à de graves complications.

Da überdies von zuverlässigen und des Landes kundigen Persönlichkeiten ebenfalls versichert wurde, daß es unmöglich sei, in ägyptischen Dörfern Leichen zur Sektion zu bekommen, so mußte darauf verzichtet werden, dem Lauf der Cholera Nil aufwärts zu folgen.

Auch in Syrien scheint die Cholera gegen alle Erwartung keinen Fuß gefaßt zu haben. Da die im Gange befindlichen Untersuchungen nur noch für ungefähr 2 Wochen Beschäftigung verschaffen können, so werden die Arbeiten wegen Mangel an geeignetem Material alsdann vorläufig unterbrochen werden müssen.

Die Kommission ist aber von dem lebhaften Wunsche beseelt, das begonnene Werk fortzusetzen und womöglich auch die ihr gestellte Aufgabe zụ lösen. Sie würde es schmerzlich empfinden, wenn die bis jetzt gewonnenen Resultate fruchtlos bleiben sollten.

Die einzige Möglichkeit zur Fortsetzung der Untersuchung bietet sich zurzeit in Indien, wo in mehreren großen Städten, insbesondere in Bombay, die Cholera noch in einem Umfange herrscht, daß ein baldiges Aufhören derselben nicht zu erwarten ist. Auch würde sich dort unzweifelhaft der Anschluß an ein Hospital, welcher sich in Alexandrien so sehr vorteilhaft erwiesen hat, am ehesten bewerkstelligen lassen. Ew. Exzellenz hochgeneigtem Ermessen stelle ich demgemäß ganz gehorsamst anheim, ob unter den obwaltenden Verhältnissen die Fortsetzung der Untersuchungen in Indien statthaben soll, und stelle ich mich, wenn Ew. Exzellenz für die Ausdehnung der Expedition nach Indien sich hochgeneigtest entschließen, zur Führung derselben auch ferner ganz gehorsamst zur Verfügung. Auch die beiden ärztlichen Mitglieder, der Expedition, die Stabsärzte Herr Dr. G a f f k y und Herr Dr. Fi s c h e r, sind bereit, sich an einer derartigen weiteren Expedition zu beteiligen. Auf die Hilfe des Chemikers Herrn T r e s k o w, welche bei der Einrichtung und dem bisherigen Betriebe des Laboratoriums unentbehrlich war, würde ich für diesen Fall Verzicht leisten können. Ganz gehorsamst habe ich noch über weitere Arbeiten, welche die Kommission neben ihren Untersuchungen über die Cholera auszuführen Gelegenheit fand, zu berichten.

Ägypten ist sehr reich an parasitischen und ansteckenden Krankheiten, und es fiel daher nicht schwer, teils zum kontrollierenden Vergleich mit den bei der Cholera gewonnenen Resultaten, teils um über wichtige die Infektionskrankheiten betreffende allgemeine Fragen weitere Aufschlüsse zu gewinnen, geeignete Untersuchungsobjekte zu erhalten. 
Berichte üb. d. Tätigkeit der zur Erforschung d. Cholera usw. entsandten Kommission.

So habe ich bisher zwei Fälle von Dysenterie seziert. In dem einen, welcher akut verlaufen war, fanden sich in der erkrankten Darmschleimhaut eigentümliche Parasiten, welche nicht zur Gruppe der Bakterien gehören und bis dahin unbekannt waren.

Dann sezierte ich im arabischen Hospital einen an Darmmilzbrand gestorbenen Araber. Die Erkrankung desselben ist wahrscheinlich auf eine Infektion durch Schafe zurückzuführen, welche aus Syrien in großer Zahl nach Ägypten importiert werden und hier massenhaft an Milzbrand fallen.

Ferner bot sich die Gelegenheit im Griechischen Hospital sechs Fälle von biliösem Typhus zu beobachten, einer Krankheit, welche die größte Ähnlichkeit mit Gelbfieber besitzt, mit letzterem schon mehrfach verwechselt wurde und deswegen von größtem Interesse ist. Drei von diesen Kranken starben. Dieselben sind ebenfalls von mir seziert und sollen eingehend untersucht werden.

Außerdem sind wiederholt Untersuchungen über Mikroorganismen in der Luft und im Trinkwasser von Alexandrien angestellt.

Wenn nọch Zeit dafür zu erübrigen ist, beabsichtige ich auch über die ägyptische Augenentzündung Beobachtungen zu machen.

In bezug auf die finanziellen Verhältnisse der Expedition bemerke ich ganz gehorsamst, da $\beta$ von dem beim Generalkonsulat in Alexandrien eröffneten Kredit bis zum heutigen Tage 2000 Mark erhoben, und damit alle Anschaffungen für die Einrichtung des Laboratoriums und sämtliche für den Unterhalt der Kommission erforderlichen Ausgaben bestritten sind.

Die Arbeiten der Kommission, welche an und für sich recht anstrengend und zum größten Teil auch sehr unangenehmer Art sind, waren infolge der hohen Temperatur, welche hier herrscht, doppelt beschwerlich. Bis jetzt litt es der Gang der Untersuchungen nicht, daß sie auch nur einen Tag unterbrochen werden konnten.

Trotzdem erfreuen sich sämtliche Mitglieder bis auf geringe in den klimatischen Verhältnissen begründete und schnell vorübergehende Unpäßlichkeiten eines guten Gesundheitszustandes. Sobald eine Unterbrechung der Arbeiten zulässig ist, halte ich es indessen für notwendig, eine Erholungspause von einigen Tagen eintreten zu lassen. Vorbehaltlich der von Ew. Exzellenz hochgeneigtest zu erteilenden Genehmigung beabsichtige ich teils zum Zwecke der Erholung, teils um den Hauptkrankheitsherd der Cholera in Ägypten zu besuchen und über das Verhalten der Krankheit daselbst Nachforschungen anzustellen, die Kommission auf einige Tage nach Kairo zu führen.

Ew. Exzellenz bitte ich schließlich ganz gehorsamst über die weitere Führung der Expedition hochgeneigtest mir Instruktion erteilen zu wollen.

S u e z, den 10. November 1883.

Ưber die Tätigkeit der Kommission seit meinem letzten Bericht (dat. Alexandrien, den 17. September) habe ich folgendes zu berichten:

Trotzdem nur noch vereinzelte Cholerafälle vorkamen, fügte es der Zufall, daß noch die Sektion einer Choleraleiche im europäischen Hospital gemacht werden konnte, wobei in bezug auf das Vorkommen der Bazillen in der Darmschleimhaut derselbe Bẹfund, wie in den früheren Fällen, erhalten wurde.

Mit dem Darminhalt dieser Leiche, sowie mit den bis dahin gesammelten anderweitigen Flüssigkeiten von Cholerakranken und Choleraleichen wurden die Infektionsversuche mit den verschiedensten Modifikationen fortgesetzt. Namentlich wurde versucht, durch unmittelbare und möglichst hoch hinaufgebrachte Injektion in den 
Mastdarm der Versuchstiere, ferner durch Vermischen jener Substanzen mit Erde oder Wasser, Eintrocknen an Zeugstoffen und einige Zeit später erfolgende Verfütterung an Affen, Hunde, Mäuse und Hühner eine Infektion zu erzielen. Aber alle diese Versuche blieben ebenso wie die früheren erfolglos.

Nachdem diese Arbeiten ihren Abschluß gefunden hatten und nicht mehr zu erwarten war, daß noch weitere Gelegenheit zur Sektion von Choleraleichen sich bieten wïrde, begab sich die Kommission am 16. Oktober nach Kairo. Die Instrumente, Apparate und gesammelten pathologischen Objekte wurden, soweit sie für die Fortsetzung der Untersuchungen erforderlich waren, wohlverpackt nach Suez als Frachtgut vorausgesand.t, um von da bei der Weiterreise nach Indien mitgeführt zu werden. - Während des Aufenthaltes der Kommission in Kairo wurde von Alexandrien eine nochmalige Zunahme der Epidemie gemeldet. Doch erschien die Rückkehr nach Alexandrien nicht zweckmäßig, weil sich voraussehen ließ, daß das neue Auflodern der Epidemie nicht erheblich und nur von kurzer Dauer sein würde.

Außerdem hatten sich die Herren Dr. Schie $B$ Bey und Dr. Kartulis in Alexandrien mit sehr dankenswerter Bereitwilligkeit erboten, noch etwa vorkommendes Sektionsmaterial zu sammeln. Dies ist inzwischen geschehen, und ich habe von den genannten Herren von acht weiteren Choleraleichen die zur Untersuchung notwendigen Objekte erhalten.

Als denjenigen Platz in Indien, welcher für die Fortsetzung der Untersuchungen am meisten geeignet schien, hatte ich anfangs Bombay in Aussicht genommen, weil daselbst im August und in der ersten Hälfte des September noch zahlreiche Choleratodesfälle vorgekommen waren. Seitdem hat aber die Epidemie dort rapide abgenommen und ist anscheinend jetzt ganz erloschen. Nach dem Urteil verschiedener mit den indischen Verhältnissen vertrauter englischer Beamten wurde mir unter diesen Umständen Kalkutta als die für die Zwecke der Kommission geeignetste Stadt bezeichnet, weil daselbst die Cholera beständig mehr oder weniger herrscht. Durch diese Mitteilungen wurde ich veranlaßt, Ew. Exzellenz um die Genehmigung zur Reise der Kommission nach Kalkutta gehorsamst auf telegraphischem Wege zu bitten.

Bevor die Kommission Ägypten verließ, hielt ich es jedoch für unerläßlich, einige Fragen, welche für die Abwehr der Cholera von der größten Wichtigkeit sind, noch einem eingehenden Studium zu unterwerfen.

Es handelte sich zunächst darum, ob die von mehreren Seiten und mit großem Nachdruck aufgestellte Behauptung richtig ist, daß die diesjährige Choleraepidemie Agyptens nicht von Indien importiert, sondern im Lande selbst entstanden sei, und daß also in Zukunft in bezug auf die Produktion dieser gefährlichen Seuche Ägypten mit Indien auf die gleiche Stufe gestellt werden müsse. Um hierüber ein Urteil zu gewinnen, hat sich die Kommission noch von Alexandrien aus am 6. Oktober nach Damiette begeben, wo die Epidemie ihren Anfang gehabt hatte, und hat während mehrerer Tage dort die sorgfältigsten Untersuchungen über den Ursprung der Seuche angestellt.

Über das gewonnene Resultat behalte ich mir ausführlichen Bericht vor..

Weit wichtigere Fragen noch waren die über die. Wirksamkeit der Quarantäne und die Verschleppung der Cholera durch die nach und von Mekka gehenden Pilger. Auch hiermit hatte sich die Kommission noch während ihres Aufenthaltes in Alexandrien beschäftigt und die Einrichtungen der Quarantäneanstalten in Gabarri und Mex bei Alexandrien, sowie der an der Mündung des östlichẹn Nilarmes bei Damiette liegenden Anstalt eingehend beschäftigt.

Als aber in den letzten Wochen der Ausbruch der Cholera unter den in Mekka befindlichen Pilgern gemeldet, und die Bestimmung getroffen wurde daß die von Djeddah 
kommenden Pilger in Tor Quarantäne halten sollten, bot sich hiermit eine so überaus günstige Gelegenheit zur Information über diese wichtigen Verhältnisse, daß ich mich für verpflichtet hielt, dieselbe nicht unbenutzt vorübergehen zu lassen. - Da jedoch keine regelmäßige Verbindung mit den ägyptischen Quarantäneplätzen am Roten Meere besteht, so blieb nichts übrig, als die Vermittelung der ägyptischen Regierung in Anspruch zu nehmen, um der Kommission den Besuch der Quarantänehäfen zu ermöglichen. Auf eine vom deutschen Generalkonsulat ergangene Anfrage erbot sich Seine Hoheit der Khedive auch sofort, der Kommission den nach Tor mit Ausrüstungsgegenständen für das Quarantänelager gehenden Dampfer ,Damanhur" für jenen Zweck zur Verfügung zu stellen, ein Anerbieten, welches dankbarst angenommen wurde. Anfänglich hoffte die Kommission diese Reise in der Weise ausführen zu können, daß sie nach dem Besuch von Tor und El Wedj an der Küste des Roten Meeres südlich nach Djeddah gegangen wäre und dort den Anschluß an eine der indischen Dampferlinien erreicht hätte. Dies ging jedoch nicht, weil die Kommission in Djeddah sich einer längeren Quarantäne hätte unterwerfen müssen und damit zu viel Zeit verloren hätte. Sie mußte daher von El Wedj nach Suez zurückkehren, um eine Fahrgelegenheit zu finden. - Am 30. Oktober begab sich die Kommission von Kairo nach Suez, am 31. Oktober fuhr sie nach Tor, am 2. November von da nach El Wedj und kehrte am 7. November abends nach Suez zurück, nachdem sie auf dem Rückwege dem Quarantänelager der Pilger in Tor einen nochmaligen Besuch abgestattet und schließlich noch die Quarantäne an den Mosesquellen bei Suez besichtigt hatte.

Dieser Ausflug ist für die Kommission im höchsten Grade lehrreich gewesen. Es bot sich nämlich die Gelegenheit, beim ersten Besuch von Tor das für den Empfang der Pilger hergerichtete, aber noch unbelegte Quarantänelager zu sehen. An demselben Tage lief dann noch ein mit fast 500 Pilgern besetztes Dampfschiff des österreichischen Lloyd in den Hafen von Tor ein. Nach Angabe des Schiffsarztes war alles gesund an Bord. Aber beim Ausschiffen der Pilger und bei ihrer Überführung in das Zeltlager, was beides in Gegenwart der Kommission stattfand, zeigten sich schon einige Pilger schwer krank und der Cholera verdächtig, so daß sie sofort in das Quarantänelazarett geschickt werden mußten. Beim zweiten Besuch von Tor fand die Kommission noch ein zweites Pilgerschiff angekommen, dessen Pilger bereits gelandet waren. In beiden Zeltlagern war inzwischen die Cholera ausgebrochen; die Pilger des ersten Schiffes hatten drei Todesfälle, diejenigen des zweiten Schiffes einen Todesfall an Cholera und entsprechend viele Erkrankungen. Bei der Anwesenheit der Kommission im Lazarett wurden eine Choleraleiche und mehrere die charakteristischen Symptome der Krankheit bietende Kranke angetroffen. Im übrigen hat sich die Kommission bemüht, bei der Besichtigung der Quarantäneanstalten von El Wedj, Tor, bei den Mosesquellen und der Sanitätsanstalt in Suez einen möglichst tiefen Einblick in diese, für die Verschleppung der Cholera nach Europa so wichtigen. Verhältnisse zu gewinnen, und glaubt sich sowohl durch eigene Untersuchüngen als auch durch die bei den Beamten der Quarantäneanstalten und den Pilgern eingezogenen Erkundigungen in den Stand gesetzt, Ew. Exzellenz demnächst eine auf eigene Anschauung begründete und zuverlässige Beurteilung darüber liefern zu können. Erwähnt möge noch werden, daß auch die Kommission bei ihrer Rückkehr nach Suez mitsamt den Reiseeffekten eine Desinfektionsprozedur durchmachen mußte.

Neben diesen unmittelbar mit der Cholera sich beschäftigenden Untersuchungen hat die Kommission ihre Forschungen über die damit im Zusammenhange stehenden Fragen, wie Wasserversorgung und Filtration des Wassers, Einfluß des Fallens und Steigens des Nils auf den Gang der Epidemie, Begräbniswesen, Verunreinigung des Bodens durch Latrinen, meteorologische Verhältnisse usw. fortgesetzt. 
Außerdem wurden in Alexandrien noch zahlreiche Sektionen gemacht und dabei wertvolle Beobachtungen gesammelt über Dysenterie, über das Vorkommen von Tuberkulose in Ägypten, ferner über Parasiten, welche im Blute der Pfortader leben (Distomum haematobium) und einen sehr häufigen Leichenbefund in Ägypten bilden. Auch bot sich Gelegenheit, noch andere wichtige durch Parasiten (Anchylostomum duodenale, Filaria sanguinis hominis) bedingte Krankheiten zu sehen.

Ferner wurden fast 50 an der ägyptischen Augenkrankheit leidende Patienten untersucht und gefunden, daß mit dem. Namen dieser Krankheit zwei verschiedene Krankheitsprozesse belegt werden. Der eine, welcher bösartiger verläuft, ist durch eine Bakterienart veranlaßt, welche den Gonorrhoemikrokoklen gleicht. und höchst wahrscheinlich damit identisch ist. Bei dem zweiten, weniger gefährlichen Prozesse finden sich regelmäßig in den Eiterkörperchen sehr kleine Bazillen.

Die Rinderpest ist in Unterägypten in den letzten Monaten noch fortwährend, wenn auch nur vereinzelt, vorgekommen. Die Kommission hat sich infolgedessen vielfach bemüht, auch diese Krankheit aus eigener Anschauung kennen zu lernen. Leider waren aber alle Versuche, rinderpestkranke Tiere oder deren Kadaver zu erhalten, vergeblich.

Bei der Abreise aus Ägypten fühle ich mich verpflichtet, im Namen der Kommission die Umsicht und Sachkenntnis, mit welcher der Vertreter des deutschen Generalkonsulats die Kommission bei jeder Gelegenheit unterstützt hat, in dankbarster Anerkennung hervorzuheben. Auch die ägyptische Regierung, für welche sich anfangs weniger Gelegenheit bot, der Kommission für die Erreichung ihrer Zwecke förderlich zu sein, hat sich für die Untersuchungen über die Entstehung der Cholera in Damiette und für das Studium der Quarantäneanstalten seitens der Kommission lebhaft interessiert und diese Arbeiten durch an ihre Behörden gerichtete Empfehlungen in jeder Beziehung unterstützt.

Ganz besonders fühlt sich aber die Kommission noch Seiner Hoheit dem Khedive dafür zum aufrichtigsten Danke verpflichtet, daß derselbe ihr die Gelegenheit zu dem so sehr wichtigen Besuche der ägyptischen Quarantänehäfen gewährt hat.

K a lkutta, den 16. Dezember 1883.

Die mit der Untersuchung ïber Cholera beauftragte Kommission ist am 11. Dezember in Kalkutta eingetroffen. Die Ankunft derselben erfolgte gerade beim Abgange der Post, so daß diese Meldung erst mit der nächsten, acht Tage später abgehenden Post geschehen konnte. Hierdurch ist es allerdings auch ermöglicht, Ew. Exzellenz bereits über den Beginn der Tätigkeit der Kommission in Kalkutta berichten zu können.

Die Kommission reiste am 13. November mit dem englischen Dampfer „Clan Buchanan" von Suez ab und erreichte Kalkutta am 11. Dezember. Das Schiff hatte in Kolombo einen Aufenthalt von 31/2 Tagen und in Madras von fast 2 Tagen. Diese Gelegenheit hat die Kommission benutzt, um sich über die sanitären Verhältnisse dieser Orte, sowie über ihr Verhalten zur Cholera zu informieren, soweit dies bei der kurzen Dauer des Aufenthaltes möglich war. In Kolombo wurde keine Cholera angetroffen; den erhaltenen Mitteilungen zufolge soll die Insel Ceylon überhaupt seit etwa 5 Jahren ganz frei von Cholera gewesen sein und keineswegs, wie mehrfach angenommen ist, zu den endemischen Choleraherden gehören. In Madras herrscht dagegen augenblicklich die Cholera, in der Stadt selbst anscheinend in mäßigem Grade, dagegen heftig in einigen Städten des südlichen Teiles der Präsidentschaft, hauptsächlich in Madura und Tanjore. 
In den von der Kommission besuchten Hospitälern der Stadt Madras wurden zwar keine Cholerakranke angetroffen, aber es bot sich die erwünschte Gelegenheit, die Einrichtung des Gefängnisses zu besichtigen, sowie Erkundigungen über die Wasserversorgung und Kanalisation dieser Stadt, welche in der Geschichte der Cholera eine bedeutende Rolle spielt, einzuziehen. Außerdem erhielt die Kommission von dem mit den Choleraverhältnissen durch langjährige Erfahrung vertrauten Sanitary Commissioner Dr. F u r n e ll sehr wertvolle Mitteilungen über das Verhalten der Cholera in der Präsidentschaft Madras, so daß der Aufenthalt in Madras ein für die Zwecke der Kommission sehr nützlicher war.

Bei der Ankunft in Kalkutta wurde die Kommission vom deutschen Konsul empfangen und am folgenden Tage zum Surgeon General with the Government of India Dr. J. M. C u $\mathrm{n}$ i $\mathrm{g} \mathrm{g}$ h a $\mathrm{m}$ begleitet. Dieser nahm die Kommission in sehr liebenswürdiger Weise auf und sicherte derselben die möglichste Unterstützung sowohl in bezug auf Beschaffung der erforderlichen Arbeitsräume als die Verfügung über die in die Hospitäler Kalkuttas gelangenden Cholerafälle zu. Er führte die Kommission nach dem Medical College Hospital, woselbst vorzüglich geeignete, mit Gas- und Wasserleitung versehene Arbeitsräume ausgesucht und der Kommission zur Verfügung gestellt wurden. Am 13. Dezember konnte die Einrichtung des Laboratoriums ausgeführt und, da ein Cholerafall ins Medical College Hospital eingeliefert war, auch sofort mit den Arbeiten begonnen werden. Am 14. Dezember konnte bereits die Sektion einer vom General Hospital nach dem Medical College Hospital gesandten Choleraleiche und am nächsten Tage die Sektion von zwei weiteren Choleraleichen im Sealdah Hospital vorgenommen werden. Mit dem hierdurch gewonnenen sehr reichlichen und für die in Aussicht genommenen Experimente vorzüglich geeigneten Material sind eine Anzahl Versuche in Gang gesetzt, und die Kommission befindet sich wieder in voller Tätigkeit.

Gegen Ende des November hatte die Zahl der Choleratodesfälle in Kalkutta ihr Minimum erreicht; seitdem ist sie jedoch wieder im Zunehmen begriffen, und nach dem Urteil der hiesigen Ärzte werden in der nächsten Zeit stets so viele Cholerafälle in die Hospitäler gelangen, daß es der Kommission an Untersuchungsobjekten nicht fehlen wird. Sehr wesentlich ist es auch, daß sich der Obduktion von Choleraleichen in den hiesigen Hospitälern anscheinend gar keine Schwierigkeiten entgegenstellen, und daß die Obduktionen frühzeitig genug nach dem Tode vorgenommen werden können, um durch Fäulnis bedingte Störungen in der Untersuchung auszuschließen. In Berücksichtigung allèr dieser Umstände bin ich davon überzeugt, daß in betreff des Ortes zur Fortsetzung der Untersuchungen über Cholera keine bessere Wahl getroffen werden konnte.

Die ferneren Aufgaben, welche die Kommission in Hinblick auf die Gewinnung praktisch verwertbarer Resultate zu erledigen haben wird, habe ich zusammengestellt und erlaube mir dieselben Ew. Exzellenz im nachstehenden vorzulegen.

I. Mikroskopische Untersuchung eines möglichst zahlreichen Obduktionsmaterials zur Erweiterung und zur Prüfung der in Ägypten erhaltenen Befunde über das Vorkommen von Bazillen in der Darmschleimhaut von Choléraleichen. Insbesondere auch Versuche über spezifische Eigenschaften dieser Bazillen in mikroskopischer Beziehung, um eine sichere Unterscheidung derselben von anderen, in Gestalt und Größe ähnlichen Bazillen zu gewinnen.

II. Nachforschungen über das Vorkommen von Cholera bei Tieren. Wiederaufnahme der Infektionsversuche mit Cholerastoffen an verschiedenen Tiergattungen; namentlich auch mit Methoden, welche bisher noch nicht benutzt wurden, z. B. direkte Injektion in den Darm. 
III. Gewinnung von Reinkulturen der im Darm der Choleraleichen gefundenen Bazillen und Benutzung dieser Reinkulturen zu Infektionsversuchen an Tieren.

IV. Bestimmung der biologischen Eigenschaften dieser Bazillen, insbesondere Sporenbildung, Lebensdauer, Verhalten in verschiedenen Nährmedien und bei verschiedenen Temperaturen.

V. Desinfektionsversuche, um die Bazillen im Wachstum zu behindern resp. zu vernichten.

VI. Untersuchung von Boden, Wasser und Luft in ihren Beziehungen zum Cholerainfektionsstoff, namentlich in bezug auf die Frage, ob derselbe in den endemischen Choleragebieten unabhängig vom menschlichen Körper, beispielsweise an bestimmte Zersetzungsvorgänge im Boden gebunden, existieren kann.

VII. Spezielle Nachforschungen über die Choleraverhältnisse in Indien, und zwar:

a) Zusammenhang der Cholera in den endemischen Gebieten mit besonderen Eigentümlichkeiten der daselbst lebenden Bevölkerung und ihrer Umgebung.

b) Choleraausbrüche in Gefängnissen, unter Truppen, auf Schiffen.

c) Verhältnisse der im endemischen Gebiete der Cholera am meisten heimgesuchten, sowie der von der Krankheit verschonten Plätze.

d) Art und Weise der Verschleppung der Cholera über die Grenzen des endemischen Gebietes und die Wege, auf welchen die Verschleppung sowohl in Indien, als über die Grenzen Indiens hinaus stattfindet. (Die Kommission hat hierbei besonders die Beförderung der Infektion durch gewisse religiöse Gebräuche und die Ausbreitung der Krankheit durch das Pilgerwesen im Auge, ferner die Verbreitung durch Schiffahrt und auf Handelsstraßen.)

e) Die in Indien bewährt gefundenen Maßregeln zur Verminderung der Cholera in Gefängnissen und unter den Truppen und die Bedingungen, unter denen in einigen indischen Städten, wie Madras, Pondichery, Guntur, Kalkutta, eine auffallende Abnahme der Cholerasterblichkeit stattgefunden hat.

Die Kommission beabsichtigt für den Fall, daß die Untersuchungen über die milkroskopischen Erreger der Cholera nicht zu dem Grade der Sicherheit gelangen, um praktischen Maßnahmen zugrunde gelegt werden zu können, den unter VII aufgeführten Punkten eine besondere Aufmerksamkeit zu widmen, um Ew. Exzellenz demnächst praktisch verwertbare Vorschläge zur Abhaltung resp. zur Minderung der Choleragefahr für das Deutsche Reich unterbreiten zu können.

Kalk u t ta, den 7. Januar 1884.

Ew. Exzellenz beehre ich mich im Verfolg meines Berichtes vom 16. Dezember v. J. über die Tätigkeit der Cholerakommission in Kalkutta ganz gehorsamst ferneren Bericht zu erstatten.

Die Kommission hatte sich der regen Teilnahme und besten Unterstützung seitens der hiesigen Behörden und Hospitalvorstände zu erfreuen. Fast sämtliche in den Hospitälern der Stadt zur Sektion kommenden Choleraleichen konnten für die Untersuchung verwertet werden. Bis jetzt ist von insgesamt 9 Selztionen und außerdem von 8 Cholerakranken Material gesammelt. Die einzelnen Fälle folgten in ziemlich gleichmäßigen Zeiträumen, so daß gerade hinreichend Zeit blieb, um die Untersuchung derselben nach allen Richtungen hin durchführen zu können. Mehrere Fälle, welche nach sehr kurzem Verlauf und ohne jede Komplikation mit anderen Krankheitszuständen tödlich geendet hatten, lieferten, da sie überdies sehr bald nach dem Tode seziert werden konnten, aus- 
gezeichnete Untersuchungsobjekte. Diesen günstigen Verhältnissen ist es zu verdanken, daß die Kommission bereits wesentliche Fortschritte in der Lösung der ihr gestellten Aufgabe machen konnte.

Zunächst bestätigte die mikroskopische Untersuchung auch in allen diesen Fällen das Vorhandensein derselben Bazillen im Choleradarm, wie sie in Ägypten gefunden waren. In meinem gehorsamsten Bericht vom 17. September v. J. mußte ich es indessen noch unentschieden lassen, ob diese Bazillen nicht wie so viele andere Bakterien zu den regelmäßigen Parasiten des menschlichen Darmes gehören und nur unter dem Einflusse des Krankheitsprozesses der Cholera in die Schleimhaut des Darmes einzudringen vermögen. Es fehlte damals noch an Merkmalen, um diese Bazillen von sehr ähnlich geformten anderen Darmbazillen unterscheiden zu können. Dieser Mangel ist nun aber glücklicherweise beseitigt. Denn mit Hilfe der im Gesundheitsamte ausgebildeten $\mathrm{Me}$ thoden, welche sich auch bei dieser Gelegenheit vorzüglich bewährt haben, gelang es, aus dem Darminhalt der reinsten Cholerafälle die Bazillen zu isolieren und in Reinkulturen zu züchten. Die genaue Beobachtung der Bazillen in ihren Reinkulturen führte dann zur Auffindung von einigen sehr charakteristischen Eigenschaften bezüglich ihrer Form und ihres Wachstums in Nährgelatine, wodurch sie mit Sicherheit von anderen Bazillen zu unterscheiden sind. Damit waren nun aber die Mittel an die Hand gegeben, um die Frage definitiv zu entscheiden, ob diese Bazillen zu den gewöhnlichen Bewohnern des Darmes gehören, oder ob sie ausschließlich im Darm der Cholerakranken vorkommen.

Zuerst wurden mit Hilfe der Gelatinekulturen ebenfalls die Bazillen in den Dejektionen der Cholerakranken und im Darminhalt der Choleraleichen nachgewiesen, und zwar gelang dies in sämtlichen hier untersuchten Fällen. Dann aber wurde der Darminhalt anderer Leichen in gleicher Weise untersucht, und es stellte sich heraus, daß die Bazillen des Choleradarmes stets fehlten. Bis jetzt sind 8 Leichen von an Pneumonie, Dysenterie, Phthisis, Nierenleiden Verstorbenen untersucht. Ferner wurde der Darminhalt von verschiedenen Tieren, sowie andere bakterienreiche Substanzen darauf geprüft, aber bislang nirgendwo den Cholerabazillen gleichende Bakterien angetroffen. Wenn sich dieser Befund auch im weiteren Verlaufe als ein ganz konstanter herausstellen sollte, dann wäre damit ein sehr wichtiges Resultat gewonnen. Denn wenn diese mit spezifischen Eigenschaften begabten Bazillen ganz ausschließlich dem Choleraprozeß angehören, dann würde der ursächliche Zusammenhang zwischen dem Auftreten dieser Bakterien und dem Choleraprozeß kaum noch einem Zweifel unterliegen können, selbst wenn die Reproduktion der Krankheit an Tieren nicht gelingen sollte. Aber auch in dieser letzteren Beziehung scheinen sich die Verhältnisse günstig zu gestalten, da in letzter Zeit einige der mit Tieren angestellten Experimente Resultate gegeben haben, welche weitere Erfolge hoffen lassen.

Neben diesen Arbeiten hat sich die Kommission noch damit beschäftigt, sich über das höchst interessante und wichtige Verhalten der Cholera in der Stadt Kalkutta möglichst zu informieren. In Städten außerhalb Indiens, welche nur in längeren Zeiträumen der Cholerainfektion ausgesetzt sind, kann der Einfluß, welchen sanitäre Verbesserungen, z. B. Zufuhr von gutem Trinkwasser, Bodendrainage und dergleichen, auf die Cholera ausüben, nicht mit Sicherheit bestimmt werden, da das einmalige oder selbst wiederholte Verschontbleiben eines solchen Ortes immer noch durch Zufälligkeiten bedingt sein kann. Dagegen muß in Städten, welche wie Kalkutta alljährlich eine beträchtliche Choleramortalität haben, jede Maßregel, welche der Cholera erfolgreich entgegenwirkt, eine mehr oder weniger bemerkbare und andauernde Herabsetzung der Mortalitätsziffer zur Folge haben. Nun hat aber in Kalkutta in der Tat seit dem Jahre 1870 die Cholera plötzlich in ganz auffallender Weise abgenommen. Vor 1870 war die alljährliche Cholera- 
sterblichkeit in Kalkutta durchschnittlich 10,1 auf 1000 Einwohner. Seit 1870 ist sie auf 3, also um mehr als das Dreifache herabgegangen. Es ist dies eine Tatsache, welche die höchste Beachtung verdient und zu Fingerzeigen für die erfolgreiche Bekämpfung der Krankheit führen muß. Nach dem fast einstimmigen Urteil der hiesigen Ärzte ist die Abnahme der Cholera allein der Einführung einer Trinkwasserleitung zuzuschreiben. Es wird eine wichtige Aufgabe der Kommission sein, hierüber durch eigene Anschauung und eigenes Studium ein selbständiges Urteil zu gewinnen. Zu diesem Zwecke hat die Kommission sowohl die Wasserwerke als auch die Kanalisationseinrichtungen von Kalkutta besichtigt. Auch ist eine Anzahl Proben des Flußwassers vor und nach der Filtration in den Wasserwerken von Pultah untersucht und das der Stadt zugeführte Trinkwasser als · von vorzüglicher Beschaffenheit gefunden.

Aus medizinischen Zeitschriften habe ich ersehen, daß die zur Erforschung der Cholera nach Ägypten gesandte französische Kommission in dem von ihr erstatteten Berichte angibt, zu anderen Resultaten, als den von mir gehorsamst gemeldeten gelangt $\mathrm{zu}$ sein und im Blute Organismen gefunden zu haben, welche der Cholera eigentümlich sein sollen. Es könnte hiernach scheinen, daß die deutsche Kommission sich in ihren Forschungen auf einem falschen Wege befindet, und ich halte es deswegen für geboten, Ew. Exzellenz ganz gehorsamst meine Ansichten über jene Angaben darzulegen.

Es kommen im Blute des gesunden Menschen neben roten und weißen Blutliörperchen kleine rundliche blasse Formelemente, die sogenannten Blutplättchen, in wechselnder Zahl vor. In manchen fieberhaften Krankheiten, z. B. Flecktyphus, Pneumonie, sind diese Gebilde sehr vermehrt, und sie sind wegen ihrer Ähnlichkeit mit Mikroorganismen schon mehrfach für Bakterien gehalten. Auch im Blute der Cholerakranken und Choleraleichen sind sie fast regelmäßig vermehrt, wie wir in den von uns untersuchten Cholerafällen ebenfalls konstatieren konnten. Diese Tatsache ist übrigens nicht neu, sondern bereits von früheren Forschern erwähnt. Beispielsweise ist von D. D. $\mathrm{Cu} n \mathrm{ningham}$ in seiner Schrift: ,Microscopical and physiological researches into the nature of the agent producing cholera" schon im Jahre 1872 eine recht gute Abbildung dieser Formelemente des Cholerablutes gegeben. Da nun selbst die bewährtesten Untersuchungsmethoden im Cholerablute keine anderen Gebilde erkennen lassen, welche balkterienähnlich sind, und da die von der französischen Kommission gegebene Beschreibung auf die erwähnten Blutplättchen in jeder Beziehung paßt, so kann ich nicht anders annehmen, als daß die französische Kommission in denselben Irrtum wie vor ihr andere Forscher gefallen ist und die Blutplättchen für spezifische Organismen gehalten hat. Irgend einen ätiologischen Zusammenhang mit der Cholera können diese Blutplättchen schon aus dem Grunde nicht haben, weil sie, wie bereits erwähnt ist, auch im Blute gesunder und solcher Menschen vorkommen, welche an anderen Krankheiten leiden.

Ka l k u t ta, den 2. Februar 1884.

Die in meinem letzten Berichte vom 7. Januar cr. noch unentschieden gelassene Frage, ob die im Choleradarm gefundenen Bazillen ausschließlich der Cholera angehörige Parasiten sind, kann nunmehr als gelöst angesehen werden.

Es war anfangs außerordentlich schwierig wegen der ungleichen Verhältnisse, unter welchen die pathologischen Veränderungen im Choleradarm sich darbieten, und wegen der großen Zahl der stets im Darm vorhandenen Bakterien das Richtige herauszufinden. In den meisten Fällen erfolgt nämlich der Tod nicht auf der eigentlichen 
Höhe des Choleraprozesses, sondern in der sich unmittelbar daran schließenden Reaktionsperiode, in welcher so bedeutende Veränderungen in der Beschaffenheit des Darmes und seines Inhaltes eintreten, daß es unmöglich ist, aus solchen Fällen allein eine klare Vorstellung von dem Choleraprozeß zu gewinnen. Erst wenn man eine Anzahl von unkomplizierten Fällen zu sezieren und frische Erkrankungsfälle damit zu vergleichen Gelegenheit gehabt hat, gelingt es, einen richtigen Einblick in die pathologischen Verhältnisse der Cholera zu gewinnen. Aus diesem Grunde war es geboten, in der Deutung der in bezug auf die Cholerabakterien erhaltenen Befunde die größte Vorsicht walten $\mathrm{zu}$ lassen und so lange mit einem bestimmten Urteil über ihr kausales Verhältnis zur Cholera zurückzuhalten, bis die volle Überzeugung davon gewonnen war.

Im letzten Berichte konnte ich bereits gehorsamst mitteilen, daß an den Bazillen des Choleradarmes besondere Eigenschaften aufgefunden wurden, durch welche sie mit aller Sicherheit von anderen Bakterien zu unterscheiden sind. Von diesen Merkmalen sind folgende die am meisten charakteristischen: Die Bazillen sind nicht ganz geradlinig, wie die übrigen Bazillen, sondern ein wenig gekrümmt, einem Komma ähnlich. Die Krümmung kann mitunter sogar soweit gehen, daß das Stäbchen fast eine halbkreisförmige Gestalt annimmt. In den Reinkulturen entstehen aus diesen gekrümmten Stäbchen oft s-förmige Figuren und mehr oder wenige lange, schwach wellenförmig gestaltete Linien, von denen die ersteren zwei Individuen und die letzteren einer größeren Zahl der Cholerabazillen entsprechen, die bei fortgesetzter Vermehrung im Zusammenhange geblieben sind. Sie besitzen außerdem Eigenbewegung, welche sehr lebhaft und am besten in einem am Deckglase suspendierten Tropfen Nährlösung zu beobachten ist; in einem solchen Präparat sieht man die Bazillen mit großer Geschwindigkeit nach allen Richtungen durch das mikroskopische Gesichtsfeld schwimmen.

Ganz besonders charakteristisch ist ihr Verhalten in Nährgelatine, in welcher sie farblose Kolonien bilden, welche anfangs geschlossen sind und so aussehen, als ob sie aus stark glänzenden kleinen Glasbrocken zusammengesetzt sind. Allmählich verflüssigen diese Kolonien die Gelatine und breiten sich dann bis zu einem mäßigen Umfange aus. In Gelatinekulturen sind sie daher durch dies eigentümliche Aussehen mit großer Sicherheit mitten zwischen anderen Bakterienkolonien zu erkennen und können von diesen auch leicht isoliert werden. Außerdem lassen sie sich auch ziemlich sicher durch die Kultur in hohlen Objektträgern nachweisen, da sie sich immer an den Rand des Tropfens der Nährflüssigkeit begeben und daselbst in ihren eigentümlichen Bewegungen und nach Anwendung von Anilinfarblösungen an der kommaähnlichen Gestalt erkannt werden können.

Bis jetzt sind 22 Choleraleichen und 17 Cholerakranke in Kalkutta zur. Untersuchung gelangt. Alle diese Fälle wurden sowohl mit Hilfe der Gelatinekulturen, als auch in mikroskopișchen Präparaten, meisten's zugleich auch noch durch die Kulturen in hohlen Objektträgern auf das Vorhandensein der spezifischen Bakterien geprüft, und ausnahmslos konnten die kommaähnlichen Bazillen nachgewiesen werden.

Dieses Resultat, zusammengenommen mit dem in Ägypten erhaltenen, berechtigt zu dem Schlusse, daß diese Bakterienart regelmäßig im Choleradarm vorkommt.

Zur Kontrolle wurden dagegen ganz in derselben Weise untersucht: 28 andere Leichen (davon 11 Dysenterien), ferner Ausleerungen eines Falles von einfacher Diarrhöe, von Dysenterie und von einem Gesunden nach überstandener Cholera, dann noch verschiedene gesunde, sowie an Darmgeschwüren und Pneumonie gestorbene Tiere, schließlich auch mit putriden Massen verunreinigtes Wasser (verschiedene Proben von städtischer Spüljauche, Wasser aus stark verunreinigten Sümpfen, Sumpfschlamm, unreines Flußwasser). Es gelang aber nicht ein einziges Mal, weder im Magen oder Darm der Menschen 
oder Tierleichen, noch in den Ausleerungen oder in den an Bakterien überaus reichen Flüssigkeiten die Cholerabazillen nachzuweisen, Da durch Arsenilkvergiftung ein der Cholera sehr ähnlicher Krankheitsprozeß bewirkt werden kann, so wurde auch ein solcher Versuch angestellt, und ein Tier nach Arsenikvergiftung auf das Vorkommen der Kommabazillen in den Verdauungsorganen geprüft, aber ebenfalls mit negativem Erfolge.

Aus diesen Resultaten ist nụn weiter der Schluß zu ziehen, daß die kommaähnlichen Bazillen ganz allein der Cholera eigentümlich sind.

Was nun das Verhältnis dieser Bakterien zur Cholera betrifft, so kamn dasselbe, wie in einem früheren Berichte bereits gehorsamst auseinandergesetzt wurde, entweder ein derartiges sein, daß diese spezifische Art von Bakterien in ihrem Wachstum durch den Choleraprozeß lediglich begünstigt wird und sich deswegen in so auffallender Weise mit der Cholera kombiniert, oder daß die Bakterien die Ursache der Cholera sind und die Krankheit nur dann entsteht, wenn diese spezifischen Bakterien ihren Weg in den Darm des Menschen gefunden haben. Die erstere Annahme ist indessen aus folgenden Gründen nicht zulässig. Es muß nämlich vorausgesetzt werden, daß ein Mensch, wenn er cholerakrank wird, diese Art von Bakterien bereits in seinem Verdauungskanal hat, und daß ferner, da diese besonderen Bakterien sowohl in Ägypten als auch in Indien, zwei ganz getrennten Ländern, in einer verhältnismäßig großen Zahl von Fällen ausnahmslos konstatiert wurden, überhaupt jeder Mensch dieselben besitzen muß. Dies kann aber nicht der Fall sein, denn, wie bereits angeführt wurde, sind die kommaähnlichen Bazillen niemals außer in Cholerafällen gefunden.

Selbst bei Darmaffektionen, wie Dysenterie und Darmkatarrh, zu welchen die Cholera besonders häufig hinzutritt, fehlten sie. Auch ist zu berücksichtigen, daß, wenn diese Bakterien so regelmäßig im menschlichen Körper vorhanden wären, sie doch gewiß schon früher das eine oder andere Mal beobachtet wären, was ebenfalls nicht der Fall ist.

Da also die Vegetation dieser Bakterien im Darm nicht durch die Cholera bewirkt sein kann, so bleibt nur noch die zweite Annahme übrig, daß sie die Ursache der Cholera sind. Daß dies aber auch in der Tat so ist, dafür spricht noch eine Anzahl anderer Tatsachen in untrüglicher Weise. Vor allem ihr Verhalten während des Krankheitsprozesses. Ihr Vorkommen beschränkt sich auf dasjenige Organ, welches der Sitz der Krankheit ist, auf den Darm. Im Erbrochenen konnten sie bisher nur zweimal nachgewiesen werden, und in beiden Fällen ließ das Aussehen und die alkalische Reaktion der erbrochenen Flüssigkeit erkennen, daß Darminhalt und mit diesem die Bakterien in den Magen gelangt waren. Im Darm selbst verhalten sie sich folgendermaßen. In den ersten Ausleerungen der Kranken finden sich, solange sie noch eine fäkulente Beschaffenheit haben, nur wenige Cholerabazillen; die dann folgenden wässerigen, geruchlosen Ausleerungen dagegen enthalten die Bazillen in großer Menge, während dann gleichzeitig alle übrigen Bakterien fast vollkommen verschwinden, so daß die Cholerabazillen in diesem Stadium der Krankheit nahezu eine Reinkultur im Darm bilden. Sobald der Choleraanfall aber abnimmt und die Ausleerungen wieder fäkulent werden, verschwinden die kommaähnlichen Bakterien in den Ausleerungen allmählich wieder und sind nach dem vollständigen Überstehen der Krankheit überhaupt nicht mehr zu finden. Ganz ähnlich ist auch der Befund in den Choleraleichen. Im Magen wurden keine Cholerabazillen angetroffen. Der Darm verhielt sich verschieden, je nachdem der Tod noch während des eigentlichen Choleraanfalls oder nach demselben eingetreten war. In den frischesten Fällen, in denen der Darm eine gleichmäßige hellrote Färbung zeigt, die Schleimhaut noch frei von Blutergüssen ist und der Darminhalt aus einer weißlichen geruchlosen Flüssigkeit besteht, finden sich die Cholerabazillen im Darm in ganz enormen Massen und nahezu rein. Thre Verteilung entspricht ganz genau dem 
Grade und der Ausbreitung der entzündlichen Reizung der Darmschleimhaut, indem sie gewöhnlich im oberen Teile des Darmes nicht so zahlreich sind, aber nach dem unteren Teile des Dünndarmes hin zunehmen. Tritt dagegen der Tod später ein, dann finden sich die Zeichen einer bedeutenden Reaktion im Darm. Die Schleimhaut ist dunkel gerötet, im unteren Teile des Dünndarmes von Blutextravasaten durchsetzt und oft in den oberflächlichsten Schichten abgestorben. Der. Darminhalt ist in diesem Falle mehr oder weniger blutig gefärbt und infolge der nun wieder eintretenden massenhaften Entwicklung von Fäulnisbakterien von putrider Beschaffenheit und stinkend. Die Cholerabakterien treten in diesem Stadium im Darminhalt immer mehr zurück, sind aber in den schlauchförmigen Drüsen und oft auch in deren Umgebung noch eine Zeitlang ziemlich reichlich vorhanden, ein Umstand, der zuerst auf das Vorkommen dieser eigentümlichen Bakterien im Darm der ägyptischen Cholerafälle aufmerksam gemacht hatte. Sie fehlen nur in solchen Fällen vollständig, welche nach überstandenem Choleraanfall an einer Nachkrankheit sterben.

Die Cholerabakterien verhalten sich also genau so wie alle anderen pathogenen Bakterien. Sie kommen ausschließlich in der ihnen zugehörigen Krankheit vor; ihr erstes Erscheinen fällt mit dem Beginn der Krankheit zusammen, sie nehmen an Zahl dem Ansteigen des Krankheitsprozesses entsprechend $\mathrm{zu}$ und verschwinden wieder mit dem Ablauf der Krankheit. Ihr Sitz ist ebenfalls der Ausbreitung des Krankheitsprozesses entsprechend, und ihre Menge ist auf der Höhe der Krankheit eine so bedeutende, daß ihre verderbliche Wirkung auf die Darmschleimhaut dadurch erklärt wird.

Es wäre allerdings noch zu wünschen, daß es gelingen möchte, mit diesen Bakterien eine der Cholera analoge Krankheit an Tieren künstlich zu erzeugen, um ihr ursächliches Verhältnis zur Krankheit auch ad oculos zu demonstrieren. Dies ist jedoch noch nicht gelungen, und es muß auch fraglich erscheinen, ob es jemals gelingen wird, weil allem Anscheine nach Tiere für die Cholerainfektion unempfänglich sind. Könnte irgendeine Tierspezies an Cholera erkranken, dann hätte dies in Bengalen, wo während des ganzen Jahres und über das ganze Land hinweg der Cholerainfektionsstoff verbreitet ist, irgend einmal in zuverlässiger Weise beobachtet werden müssen. Aber alle darauf gerichtete Erkundigungen sind negativ ausgefallen.

Dennoch kann die Beweiskraft der vorhin angeführten Tatsachen durch das Nichtgelingen des Tierexperiments nicht abgeschwächt werden. Auch bei anderen Infektionskrankheiten tritt uns dieselbe Erscheinung entgegen, so z. B. beim Abdominaltyphus und bei der Lepra, zwei Krankheiten, denen ebenfalls spezifische Bakterien zukommen, ohne daß es bisher gelungen ist, diese Krankheiten auf Tiere zu übertragen, und doch ist die Art und Weise des Vorkommens der Bakterien in diesen Krankheiten eine solche, daß unabweislich die Bakterien als die Ursache der Krankheit angesehen werden müssen. Dasselbe gilt auch von den Cholerabakterien.

Übrigens hat das weitere Studium der Cholerabakterien noch mehrere Eigenschaften derselben erkennen lassen, welche sämtlich mit dem, was über die Choleraätiologie bekannt ist, in Einklang stehen, mithin als weitere Bestätigung für die Richtigkeit der Annahme, daß die Bazillen die Choleraursache sind, dienen können.

$\mathrm{Am}$ bemerkenswertesten in dieser Beziehung ist die wiederholt gemachte Beobachtung, daß in der Wäsche der Cholerakranken, wenn sie mit den Dejektionen beschmutzt war und während 24 Stunden im feuchten Zustande gehalten wurde, die Cholerabazillen sich in ganz außerordentlicher Weise vermehrten. Es kann dieses Verhalten eine Erklärung für die bekannte Tatsache geben, daß die Cholerawäsche so häufig die Veranlassung zur Infektion solcher Personen abgibt, welche damit zu tun haben. Durch diese Beobachtung aufmerksam gemacht, wurden weitere Versuche angestellt und 
gefunden, daß dieselbe Erscheinung eintritt, wenn Choleradejektionen oder Darminhalt von Choleraleichen auf der feucht gehaltenen Oberfläche von Leinewand, Fließpapier und ganz besonders auf der Oberfläche feuchter Erde ausgebreitet wird. Nach 24 Stunden hatte sich regelmäßig die ausgebreitete dünne Schleimschicht vollständig in eine dichte Masse von Cholerabazillen verwandelt.

Eine weitere sehr wichtige Eigenschaft der Cholerabakterien ist die, daß sie nach dem Eintrocknen so rasch absterben, wie kaum eine andere Bakterienart. Gewölhnlich ist schon nach dreistündigem Trocknen alles Leben in ihnen erloschen.

Es hat sich ferner noch ergeben, daß ihr Wachstum nur in alkalisch reagierenden Nährsubstanzen regelrecht erfolgt. Schon eine sehr geringe Menge freier Säure, welche das Wachstum anderer Bakterien noch nicht merklich beeinflußt, hält sie in der Entwicklung auffallend zurück.

Im normal funktionierenden Magen werden sie zerstört, was daraus hervorgeht, daß wiederholt bei Tieren, welche anhaltend mit Cholerabazillen gefüttert und dann getötet waren, weder im Magen noch im Darmkanal die Bazillen nachgewiesen werden konnten. Diese letztere Eigenschaft zusammen mit der geringen Widerstandsfähigkeit gegen das Eintrocknen gibt eine Erklärung dafür, daß, wie es die tägliche Beobachtung lehrt, bei dem unmittelbaren Verkehr mit den Cholerakranken und deren Produkten so selten eine Infektion erfolgt. Es müssen offenbar, damit die Bazillen in den Stand gesetzt werden, den Magen zu passieren, um dann im Darm den Choleraprozeß hervorzurufen, noch besondere Umstände zu Hilfe kommen. Vielleicht können die Bazillen unbeschädigt durch den Magen gehen, wenn die Verdauung gestört ist, wofür die in allen Choleraepidemien und auch hier in Indien regelmäßig gemachte Beobachtung spricht, daß besonders häufig solche Menschen an Cholera erkranken, welche sich eine Indigestion zugezogen haben oder sonst an Verdauungsstörungen leiden. Vielleicht aber befähigt auch ein besonderer Zustand, in welchen diese Bakterien versetzt werden, und welcher dem Dauerzustande anderer Bakterien analog sein würde, dieselben, den Magen unbeschädigt passieren zu können.

Es ist allerdings nicht wahrscheinlich, daß diese Veränderung in der Produltion von Dauersporen besteht, da solche Sporen erfahrungsgemäß viele Monate, selbst Jahre lebensfähig bleiben, während sich das Choleragift nicht länger als ungefähr 3-4 Wochen wirksam erhält. Trotzdem ist es sehr wohl denkbar, daß irgendeine andere Form von Dauerzustand existiert, in welcher die Bazillen einige Wochen in getrocknetem Zustande am Leben bleiben können, und in welchem sie auch imstande sind, der zerstörenden Wirkung der Magenverdauung zu widerstehen.

Die Umwandlung in einen solchen Zustand würde dem entsprechen, was $\mathrm{P}$ e tt e n k of er als Reifung des Cholerainfektionsstoffes bezeichnet hat. Bis jetzt ist es noch nicht gelungen, einen solchen Dauerzustand der Cholerabazillen zu entdecken.

Die von den experimentellen Arbeiten nicht in Anspruch genommene Zeit hat die Kommission benutzt, um ein sehr reichhaltiges Material über die Choleraverhältnisse Indiens und speziell Bengalens, des endemischen Choleragebietes, entsprechend den in meinem gehorsamsten Bericht vom 16. Dezember v. J. unter Nr. VII. bezeichneten Punkten zu sammeln.

Außerdem wurden verschiedene für die Cholera sehr wichtige Punkte in Kallkutta und dessen nächster Umgebung besichtigt, unter denen besonders das Fort William. und das Zentralgefängnis in Alipore zu erwähnen sind. 
K a lku tta, den 4. März 1884.

Ew. Exzellenz beehre ich mich über die von der Cholerakommission erreichten weiteren Resultate gehorsamst Bericht zu erstatten.

Es ist eine auffallende Tatsache, daß die Cholera auch in ihrem endemischen Gebiet sich sehr oft an bestimmte Lokalitäten gebunden zeigt und daselbst unverkennbare und deutlich abgegrenzte Epidemien bildet. Besonders häufig werden derartig lokalisierte. kleine Epidemien in den Umgebungen der sogenannten Tanks beobachtet. Zur Erläuterung muß erwähnt werden, daß die über ganz Bengalen in unzähliger Menge verbreiteten Tanks kleine von Hütten umgebene Teiche und Sümpfe sind, welche den Anwohnern ihren sämtlichen Wasserbedarf liefern und zu den verschiedensten Zwecken, wie Baden, Waschen der Kleidungsstücke, Reinigen der Hausgeräte und auch zur Entnahme des Trinkwassers benutzt werden.

Daß bei so mannigfaltigem Gebrauch das Wasser im Tank verunreinigt wird und keine den hygienischen Anforderungen entsprechende Beschaffenheit haben kann, ist selbstverständlich. Sehr oft kommt aber hierzu noch, daß Latrinen, wenn Einrichtungen der primitivsten Art so genannt werden dürfen, sich am Rande des Tanks befinden und ihren Inhalt in den Tank ergießen, und daß überhaupt das Tankufer als Ablagerungsstätte für allen Unrat und insbesondere für menschliche Fäkalien dient. Die Tanks enthalten deswegen in der Regel ein stark verunreinigtes Wasser, und es ist unter diesen Verhältnissen erklärlich, daß die hiesigen Ärzte solche um einen Tank gruppierte Choleraepidemien mit der schlechten Beschaffenheit des Tankwassers in Zusammenhang bringen. Diese Tankepidemien sind keineswegs selten, und fast jeder Arzt, welcher eine große Erfahrung über Cholera hat, kennt eine mehr oder weniger große Zahl von Beispielen. Ich habe deswegen schon von Anfang an meine Aufmerksamkeit auf diesen Punkt gerichtet und den Sanitary Commissioner with the Government gebeten, mich davon in Kenntnis zu setzen, wenn eine solche Epidemie in leicht erreichbarer Entfernung von Kalkutta vorkommen würde. Dieser Fall ist nun in den letzten Wochen eingetreten. Aus Saheb-Bagan, zu Belliaghatta, einer der Vorstädte von Kalkutta, gehörig, wurden während weniger Tage ungewöhnlich viele Cholerafälle gemeldet. Die Erkrankungen beschränkten sich ausschließlich auf die rings um einen Tank gelegenen, von einigen hundert Personen bewohnten Hütten, und es starben von dieser Bevölkerung 17 Personen an Cholera, während in einiger Entfernung vom Tank und im ganzen zugehörigen Polizeidistrikt die Cholera zur selben Zeit nicht herrschte. Bemerkenswert ist, daß derselbe Platz in den letzten Jahren wiederholt von Cholera heimgesucht ist. - Über den Beginn und Verlauf der Epidemie wurden nun von der Kommission sorgfältige Untersuchungen angestellt, wobei sich herausstellte, daß der Tank in der gewöhnlichen Weise von den Anwohnern zum Baden, Waschen und Trinken benutzt wird, und daß auch die mit Choleradejektionen beschmutzten Kleider des ersten tödlich verlaufenden Cholerafalles im Tank gereinigt waren. Es wurde dann ferner eine Anzahl Wasserproben von verschiedenen Stellen des Tanks und zu verschiedenen Zeiten entnommen, mit Hilfe der Nährgelatinekultur untersucht, und die Cholerabazillen in mehreren der ersten Wasserproben ziemlich reichlich gefunden. Unter den späteren Proben, welche am Ende der Epidemie geschöpft waren, enthielt nur noch eine, welche von einer besonders stark verunreinigten Stelle des Tanks herstammte, die Cholerabazillen, und zwar auch nur in sehr geringer Zahl. Wenn man berücksichtigt, daß bis dahin vergeblich in zahlreichen Proben von Tankwasser, Sewage, Flußwasser und sonstigem allen Verunreinigungen ausgesetztem Wasser nach den Cholerabazillen gesucht wurde, und daß sie zum ersten Male mit allen ihren charakteristischen Eigenschaften in einem von einer Cholera- 
epidemie umschlossenen Tank gefunden sind, dann muß dies Resultat als ein höchst wichtiges angesehen werden. Es steht fest, daß das Wasser im Tank infiziert wurde durch Cholerawäsche, welche nach den früheren Beobachtungen die Cholerabazillen besonders reichlich zu enthalten pflegt; ferner ist konstatiert, daß die Anwohner des Tanks dieses infizierte Wasser zu häuslichen Zwecken und namentlich zum Trinken benutzt haben. Es handelt sich hier also gewissermaßen um ein durch den Zufall herbeigeführtes Experiment am Menschen, welches den Mangel des Tierexperimentes in diesem Falle ersetzt und als eine weitere Bestätigung für die Richtigkeit der Annahme dienen kann, daß die spezifischen Cholerabazillen in der Tat die Krankheitsursache bilden.

Bis jetzt steht dieses Faktum allerdings noch vereinzelt da, aber immerhin zeigt uns dasselbe einen der Wege, auf welchen das Choleragift in den menschlichen Körper gelangen kann, und ich zweifle nicht, daß auch in anderen ähnlichen Fällen der Nachweis der Cholerabazillen im Wasser oder sonstigen Vehikeln des Infektionsstoffes gelingen muß.

Seit meinem letzten gehorsamsten Berichte sind ferner 20 Choleraleichen und die Dejektionen von 11 Cholerakranken untersucht, und es beträgt somit die Gesamtzahl der in Indien zur Untersuchung verwerteten Fälle: 42 Choleraleichen und 28 Cholerakranke. Neue Resultate haben diese letzten Fälle allerdings nicht ergeben. Sie glichen den früheren in jeder Beziehung, namentlich auch in bezug auf das Verhalten der Cholerabazillen.

Außerdem sind noch eingehende Untersuchungen über den Einfluß verschiedener Substanzen, wie Sublimat, Karbolsäure und anderer desinfizierender Stoffe auf die Entwicklung der Cholerabazillen in Nährflüssigkeiten, ferner über das Verhalten derselben in Kohlensäure und beim Abschluß von Luft angestellt. Auch wurden die Versuche, welche dazu dienen sollten, eine Dauerform der Cholerabazillen aufzufinden, unermüdlich fortgesetzt. Doch ist bis jetzt nichts derartiges aufgefunden. Die einzige Möglichkeit, die Cholerabazillen längere Zeit lebensfähig zu erhalten, besteht darin, daß man sie vor dem Eintrocknen bewahrt. In Flüssigkeiten bleiben sie wochenlang entwicklungsfähig, und es scheint alles darauf hinzuweisen, daß sie nur in feuchtem Zustande verschleppt und dem menschlichen Körper wirksam einverleibt werden können.

Leider mußten die weiteren Untersuchungen über diesen Gegenstand wegen der in diesem Jahre schon frühzeitig eingetretenen heißen Witterung aufgegeben werden. In den letzten Wochen war die Temperatur schon so hoch, daß nur noch unter großen Schwierigkeiten im Laboratorium gearbeitet werden konnte. Aber seit einigen Tagen ist es fast unerträglich heiß geworden, und es bleibt nichts anderes übrig, als die Arbeiten vorläufig abzubrechen.

Einen umfassenden B ericht über die Tätigkeit der zur Erforschung der Choleraim Jahre 1883 nach Ägyten und Indienentsandten Kom$\mathrm{m}$ is s i o $\mathrm{n}$ hat ihr Mitglied $\mathrm{Gaffky}$, seinerzeit kaiserl. Reg.-Rat und Mitglied des Kaiserl. Gesundheitsamtes, nachträglich, unter Mitwirkung $\mathrm{K}$ o c h s, bearbeitet. Der (359 Seiten umfassende, mit Abbildungen im Text, 30 Tafeln und einem Titelbilde versehene) Bericht ist als Bd. III der „Arbeiten aus dem Kaiserlichen Gesundheitsamte“ und separat (Berlin, J. Springer, 1887) erschienen.

Der Herausgeber. 